\title{
Research on QoS Policy Deployment Approach of DiffServ Model Based on GNS3
}

\author{
Lizhen Zhao ${ }^{1, \text { a }}$, Fan Yang ${ }^{2, b}$ \\ ${ }^{1}$ Educational Technology and Computer Center Zhaoqing University,Zhaoqing, Guangdong, China \\ ${ }^{2}$ School of Computer Science,Zhaoqing University,Zhaoqing, Guangdong, China \\ asdzlz@zqu.edu.cn, byangfan9@zqu.edu.cn
}

Keywords: GNS3; DiffServ model; QoS; Network engineering

\begin{abstract}
Based on DiffServ model architecture model, GNS3 network simulator is used to build a virtual network environment; on this basis, the main QoS technology is analyzed and discussed in DifferServ model and MQC deployment is used to ensure QoS policies of low-latency operations; SLA job simulation is used to produce different types of data traffic and the main performance parameters are detected in three cases of the normal network, congestion and the implementation of QoS policies; the experimental results verify the correctness and practical feasibility of the method.
\end{abstract}

\section{Introduction}

Today's Internet is multimedia real-time network carrying IPTV, Internet TV, VOIP, OTT and other applications. Wide use of multimedia real-time services such as voice and video greatly enhances the complexity of the Internet and urgently requires better network management and QoS control more responsive to business needs, so as to provide a suitable end to end QoS guarantee [1]. From the current level of technology, the most effective way is to achieve through DiffServ model [2]. However, faced with the network running the real business, to facilitate the conduct of policy deployment and technical training of DiffServ model is not easy. First, the artificially loaded network traffic will affect the running network services when DiffServ model QoS is tested; second, the developed DiffServ model control strategies is difficult to verify the correctness and effectiveness in the network running the business; third, profound QoS theory and complex QoS configuration commands [3] makes it difficult for network management staff to grasp quickly. Thus, QoS deployment and testing of simulation DiffServ model in the virtual network environment becomes a simple and effective way for QoS technology research and training. Through the integrated application research of GNS3 Network simulator [4], DiffServ QoS model technology and Cisco IP SLA technology [5], the paper proposes a DiffServ model QoS policy deployment and testing method based on virtual network environments; it is not restricted by the practical network environment and equipment and is convenient, affordable, flexible and easy to use. It can repeat training and trial.

\section{DiffServ model and QoS technology}

DiffServ model is a QoS model proposed by IETF working group in 1998 and is a class-based QoS technology. The main QoS technology of DiffServ model includes four aspects of traffic classification and marking, traffic policing and traffic shaping, congestion management and 
congestion avoidance. Based on the location of the different routing devices in the network topology, deploying applications of QoS technology is different. For edge router, packet classification and marking operation is typically deployed to perform, and in the core router, congestion management and congestion avoidance is deployed to perform [6].

\section{Traffic Classification}

In principle, traffic classification can be conducted in each layer of the OSI reference model; commonly used traffic classification methods are three kinds of ACCESS-LIST, CLASS-MAP and NBAR. ACCESS-LIST can accurately use protocol, source address, destination address, source port, destination port and other parameters to define the classification conditions; CLASS-MAP is a more simple and convenient traffic classification method and can make classification according to the sources of traffic and packet size; NBAR (Network-Based Application Recognition) can dynamically allocate the application of TCP / UDP port number and HTTP traffic to make classification.

\section{Traffic Policing and Traffic Shaping}

When implementing the QoS policy of DiffServ model, the token bucket technology can be used to limit the user data within a certain bandwidth; when data traffic of a user exceeds the rated bandwidth, the excess traffic will not be transferred, but can only take other ways to deal with. Traffic policing refers to directly discarding the excess traffic when the user data exceeds the bandwidth limit; traffic shaping is not to discard the excess traffic when exceeding the bandwidth limit, but to wait to transmit until the next second ${ }^{[7]}$.

\section{Congestion management and congestion avoidance}

QoS of DiffServ model mainly achieves congestion management of interface through queuing technology. Common queue technology includes FIFO, PQ, CQ, WFQ, CBWFQ, LLQ and CBLLQ. Among network equipment, all the interface with the rate less than or equal to $2.048 \mathrm{Mbpsis}$ opens

WFQ queue in default. All interface with rates greater than $2.048 \mathrm{Mbps}$ opens FIFO queue in default. Congestion avoidance refers to discarding certain traffic through technical means to reduce specific service's QoS levels to avoid congestion. Commonly used technologies are Tail Drop, WRED, FRDE and so on.

\section{DiffServ model QoS policy deployment}

\section{Building virtual network environment and QoS business requirement}

In this study, GNS3 network simulator is used to build a virtual network environment; according to the architectural features of DiffServ model, a network topology shown in Figure 1 is built; R1 and R6 represent two service routers in the DS area; R2 and R5 represent two aggregation routers in the DS region; R3 and R4 represent two core routers in the DS area. In order to achieve interoperability of the two DS areas, first plan the appropriate IP subnet and allocate IP address; network routing uses the unified OSPF routing program to ensure the whole network is reachable. 10.1.1.0/24 and 10.1.2.0/24 are two subnet goals used for QoS test, including www, ftp and other services test. 


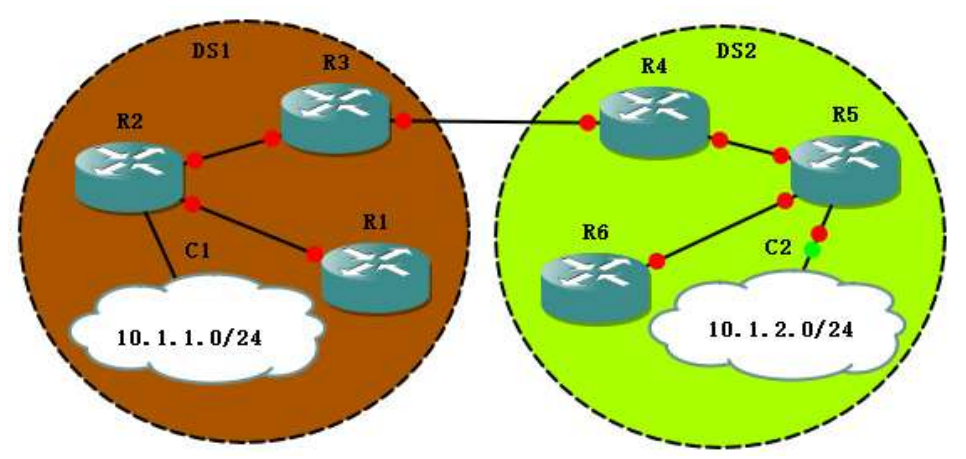

Figure 1 Network topology of DiffServ model QoS policy deployment

\section{Business performance test when network is normal}

Before deploying QoS policy, in the a virtual network environment in Figure 1, SLA operation can be used to simulate VOIP, HTTP, TELNET, DHCP, FTP, ICMP, TCP, UDP and other traffic on router R1 or R6; after running for about 20 minutes (network is stable), view the performance parameters recording various types of traffic, in order to simulate the corresponding network traffic. In VOIP jitter testing, for example configure VOIP jitter test job on router R1; test the router VOIP jitter from R1 to R5; VOIP voice connected devices of the goal router R5 should be noted; therefore, add a loopback interface in the router R5 and specify the IP address to be 9.9.9.1/24 to simulate VOIP voice connection address; use jitter operation type and configure the destination address, destination port, speech coding, packet size, test frequency and so on. Specific configuration is as follows:

\section{Conf term}

Ip sla monitor 10

Type jitter dest-ipaddr 9.9.9.1 dest-port 14280 codec g711alaw advantage-factor 1

Request-data-size 200

Frequency 10

Exit

Ip sla monitor schedule 10 life forever start-time now

\section{Business performance test in network congestion}

In real network, congestion is mainly caused due to the sudden increase in traffic. In the virtual network environment, the network function is a result of the implementation of the appropriate IOS simulation through each virtual network, so increasing the flow would mean a sharp increase in physical resources needed by each virtual device, which can easily lead to crash in network due to inadequate resources of physical host. Thus, in a virtual network environment, usually rate-limit is used to perform link speed to conduct simulate congestion. After congestion is caused, the same method is used to configure SLA operations, simulate different network traffic and test and collect the appropriate performance's operational parameters to facilitate analysis and comparison of business operational performance in different network status.

QoS policy's deployment method

In this study, MQC method is used to complete DiffServ model's QoS policy deployment. The basic process includes three links of defining traffic, setting policy and applying policy. Based on network environment in FIG. 1, assuming that the user's network application traffic mainly includes http browsing data flow, ftp file transfer data flow, VOIP data flow, telnet data flow and icmp data 
flow, QoS need of the user is to assign guaranteed bandwidth of the main business in proportion; among it, VOIP service guaranteed bandwidth is $40 \%$ of the total link bandwidth; http traffic guaranteed bandwidth is $20 \%$, ftp service guaranteed bandwidth is $10 \%$, guaranteed bandwidth of telnet, icmp and other services is $5 \%$.

As for the above QoS requirements, CBLLQ queuing policy can be selected to achieve it. Specific configuration commands are shown in Table 1.

Table 1 QoS Policy Configuration Commands

\begin{tabular}{|c|c|}
\hline (1) Traffic classification & (2) Class definition diagram \\
\hline $\begin{array}{l}\text { access }-1 \text { ist } 100 \text { permit ip any any precedence } 5 \\
\text { access }-1 \text { ist } 100 \text { permit ip any any dscp ef } \\
\text { access }-1 \text { ist } 101 \text { permit tcp any any eq www } \\
\text { access }-1 \text { ist } 102 \text { permit tcp any any eq } \mathrm{ftp} \\
\text { access }-1 \text { ist } 103 \text { permit tcp any any eq telnet } \\
\text { access-list } 103 \text { permit icmp any any }\end{array}$ & $\begin{array}{l}\text { class-map V0IP } \\
\text { match access-group } 100 \\
\text { class-map HTTP } \\
\text { match access-group } 101 \\
\text { class-map FTP } \\
\text { match access-group } 102 \\
\text { class-map ICMP-Telnet } \\
\text { match access-group } 103 \\
\end{array}$ \\
\hline (3) Configuring QoS policy figure & (4) Apply policies to the port \\
\hline $\begin{array}{l}\text { policy-map My_Qos } \\
\text { class VOIP } \\
\text { priority percent } 40 \\
\text { class HTTP } \\
\text { bandwidth percent } 20 \\
\text { class FTP } \\
\text { bandwidth percent } 10 \\
\text { class ICMP-Telnet } \\
\text { bandwidth percent } 5 \\
\text { class class-default } \\
\text { fair-queue } \\
\text { queue-limit } 60\end{array}$ & $\begin{array}{l}\text { int } \mathrm{f} 0 / 0 \\
\text { service-policy output My_Qos }\end{array}$ \\
\hline
\end{tabular}

\section{Efficacy testing and analysis}

Based on simulation network operation environment shown in Figure 1, first configure various types of SLA operations in the service router R1 to simulate different traffic of HTTP, VOIP, FTP, ICMP, TCP, UDP, etc.; collect VOIP and HTTP performance parameters in case of normal network; then, use CAR technology in the uplink port of the convergence router R2 to limit traffic; simulate running for about 10 minutes to cause mild network congestion; in this time, collect VOIP and HTTP performance parameters in case of network congestion; finally, in the uplink port of the convergence router R2, make deployment and configuration of CBLLQ based QoS policy, then collect the VOIP and HTTP parameters after QoS control policy is in effect, as shown in Table 1. After deploying QoS control strategy, VOIP and HTTP performance parameters have best performance.

Table 1 Comparison of VOIP and HTTP performance parameters in three cases of normality, congestion and QoS policy

\begin{tabular}{cccc}
\hline Test parameters & Normal (MS & Congestion (MS) & Deploy QoS (MS) \\
\hline MinofICPIF & 1 & 22 & 4 \\
MaxofICPIF & 24 & 93 & 32 \\
MinofMOS & 3.86 & 1.34 & 3.12 \\
MaxofMOS & 4.49 & 3.56 & 4.09 \\
Latest RTT & 316 & 816 & 191 \\
Latest DNS RTT & 84 & 204 & 0 \\
Latest TCP & 108 & 276 & 88 \\
Connection RTT & & 336 & 103 \\
Latest HTTP & 124 & & \\
Transaction RTT & & & \\
\hline
\end{tabular}




\section{Conclusion}

This article discusses the DiffServ model QoS policy deployment method based on virtual network environment. GNS3 network simulator is used to set up topology in line with DiffServ model QoS control; then QoS policies to ensure the low latency operations and SLA operations for network performance testing are deployed and configured; it can train the real network DiffServ model QoS configuration and performance testing technology, which greatly reduces the cost of network management and the network professional training. Since the method uses network simulator to simulate the real network, therefore, it has a higher performance requirement for the host running the simulation software; experimental results will vary depending on the host's performance, and it may be different from performance test results in real network, but the methods and conclusions are the same.

\section{Reference}

[1] Xu Hang. The design and application of QoS technology in IP bearer network [D], [Master's Thesis of Beijing University of Posts and Telecommunications], Beijing: Beijing University of Posts and Telecommunications, 2009,11.

[2] DiffServ [EB / OL], http: //baike.baidu.com/link url = OoYUOaL86wNPqWwAsy0TuYogelU cBevcleNftgf7O2edK0fMge3iy 8itw-lRNSwkQzl JhQkWVcGLGP18pEs6K_?

[3] Quality of Service Commands [EB / OL]. Http://www.cisco.com/c/en/us/td/docs/routers/crs /software/crs_r4-1/ qos / command / reference / qos_cr41crs_chapter1 .html, 2014-11-20

[4] Introduction to GNS3 [EB / OL]. Http://www.gns3.net/gns3-introduction/,2012-11-20

[5] IP SLAs Configuration Guide [EB / OL] .http: //www.cisco.com/c/en/us/td/docs/ios-xml/ios/ips la/ configuration / 15-mt / sla-15 -mt-book / sla_dns.html \# GUID-5B326F06-6E4E-4F69-A5B7BDA80C4B4996,2014-11-10

[6] Yi Jianxun, Jiang Lalin and Shi Zhangqiong. Computer network design [M]. Beijing: People's Posts and Telecommunications Press, 2011: 116-130

[7] CCNP QoS [EB / OL] .http:? //wenku.baidu.com/link Url = AQY4BAMzM7f6-07Q6F2RaADI mLOJ6gJGMZshePOmXrGZo4SHcAVQldp1s0ViUgvPNm7Jzus42czIJmVZEaawFkB7rjodNLEn kTQ-W_bEAQu 